\title{
Actin polymerization is reduced in the anterior cingulate cortex of elderly patients with schizophrenia
}

\author{
Hriday P. Bhambhvani ${ }^{1}$, Toni M. Mueller ${ }^{1}$, Micah S. Simmons ${ }^{1}$ and James H. Meador-Woodruff ${ }^{1}$
}

\begin{abstract}
Recent reports suggest abnormalities in the regulation of actin cytoskeletal dynamics in schizophrenia, despite consistent evidence for normal actin expression. We hypothesized that this may be explained by changes in the polymerization state of actin, rather than in total actin expression. To test this, we prepared filamentous actin (F-actin, polymeric) and globular actin (G-actin, monomeric) fractions from postmortem anterior cingulate cortex from 16 patients with schizophrenia and 14 comparison subjects. Additionally, binding of fluorescently-labeled phalloidin, a selectively F-actin-binding peptide, was measured in unfractionated samples from the same subjects. Western blot analysis of fractions revealed decreased F-actin, increased G-actin, and decreased ratios of F-actin/total actin and Factin/G-actin in schizophrenia. Decreased phalloidin binding to F-actin in parallel experiments in the same subjects independently supports these findings. These results suggest a novel aspect of schizophrenia pathophysiology and are consistent with previous evidence of reduced dendritic spine density and altered synaptic plasticity in schizophrenia, both of which have been linked to cytoskeletal abnormalities.
\end{abstract}

\section{Introduction}

While the etiology of schizophrenia is not established, a consistent finding in schizophrenia brain is reduced dendritic spine density and altered spine morphology ${ }^{1-4}$. The cause of these dendritic spine abnormalities is unknown. One hypothesis implicates dysfunction of the actin cytoskeleton, the stabilization of which is essential for maintaining dendritic spine morphology and density ${ }^{5}$. Consistent with this hypothesis, many proteins crucial to stabilization of the actin cytoskeleton have been shown to be abnormally expressed in schizophrenia, including reelin, fragile $\mathrm{X}$ mental retardation protein, disrupted in schizophrenia-1 (DISC-1), cell division cycle protein 42 (CDC42), and Duo ${ }^{6-9}$. These abnormalities are intriguing, however, given multiple reports of normal actin protein expression in schizophrenia brain ${ }^{10,11}$. The discrepancy may be explained by altered actin polymerization,

\footnotetext{
Correspondence: Micah S Simmons (micahsimmons@uabmc.edu)

${ }^{1}$ Department of Psychiatry and Behavioral Neurobiology, University of Alabama at Birmingham, 1719 6th Avenue South, CIRC 593A, Birmingham, AL 35294,
} USA abnormal binding of actin to cytoskeletal substrates such as cofilin and N-WASP, or abnormal turnover and restructuring of the actin cytoskeleton. Actin remodeling is known to drive both the formation and loss of dendritic spines, as well as regulate their morphological plasticity ${ }^{12}$, ${ }^{13}$. Underlying this rearrangement of actin is the dynamic polymerization of actin from its monomeric form (Gactin) to a filamentous polymer (F-actin), both of which are present in spines and affect spine morphology $y^{14}$. Interestingly, it has been hypothesized that diminished cortical dendritic spine density and the resulting reduction in excitatory projections to the ventral mesencephalon, the location of the dopamine cells that project to striatum and limbic areas, gives rise to a hyperdopaminergic state in schizophrenia ${ }^{15}$. Indeed, reduction of actin polymerization via deletion of the actin related protein-2/ 3 (Arp2/3) complex in mice induced a loss of dendritic spines and elevated striatal dopaminergic neurotransmission ${ }^{16}$.

Proteins involved in pathways regulating actin polymerization have been found to be abnormally expressed 
in schizophrenia. The Arp2/3 complex is a chief initiator of actin polymerization and regulates the formation of branched actin networks ${ }^{17}$. Previously, we reported reduced protein expression of cortactin, a nucleation promoting factor (NPF) and activator of the Arp2/3 complex $^{18}$. Additionally, reduced gene expression of cortactin, CYFIP1 (NPF), N-WASP (NPF and activator of the Arp2/3 complex), Arp2, and Arp3 was recently reported ${ }^{19}$. Altogether, these data suggest impaired actin polymerization in schizophrenia. We hypothesized that, although total actin expression is unchanged in schizophrenia, the polymerization of actin may be decreased. To test this hypothesis, we applied an F-actin/G-actin fractionation technique used frequently in cell culture studies to postmortem human brain. F-actin and G-actin enriched fractions of anterior cingulate cortex from schizophrenia and comparison subjects were probed for actin using western blot analysis to determine actin polymerization status. In the same subjects, fluorescentlylabeled phalloidin, a selectively F-actin-binding peptide, was used as an independent measure of F-actin.

\section{Methods}

\section{Subjects acquisition and tissue processing}

Samples from the full thickness of the gray matter of anterior cingulate cortex (ACC, BA24/32) of 16 schizophrenia and 14 comparison subjects (Table 1) were obtained from the Mount Sinai Medical Center brain collection, as previously described ${ }^{20}$. The diagnosis of schizophrenia was determined by two independent clinicians using DSM-III-R criteria ${ }^{21}$. All patients had a history of psychotic symptoms prior to the age of 40 and at least 10 years of hospitalization. Patients were recruited prospectively and underwent extensive antemortem clinical assessment. Subject medical history was assessed for alcoholism, substance abuse, death by suicide, coma for more than $6 \mathrm{~h}$ before death, or evidence of neurodegenerative disorders, and subjects were excluded if any of

Table 1 Summary of subject demographics

\begin{tabular}{llll}
\hline & Comparison & Schizophrenia & $\mathbf{p}$ \\
\hline$n$ & 14 & 16 & \\
Age & $79.4 \pm 9.3$ & $75.8 \pm 11.9$ & 0.36 \\
Sex & $4 \mathrm{M} / 10 \mathrm{~F}$ & $11 \mathrm{M} / 5 \mathrm{~F}$ & 0.03 \\
PMl (hours) & $10.0 \pm 7.3$ & $11.4 \pm 4.4$ & 0.54 \\
Tissue pH & $6.3 \pm 0.2$ & $6.4 \pm 0.3$ & 0.28 \\
On/Off Rx & $0 / 14$ & $11 / 5$ & \\
\hline
\end{tabular}

Values are expressed as means \pm standard deviation. Off Rx indicates patients who had not received antipsychotic medications for 6 weeks or more prior to time of death

$M$ male, $F$ female, $P M I$ postmortem interval, $R x$ antipsychotic medication these were present. Comparison subjects were assessed similarly and confirmed to be without neuropathological evidence of neurodegenerative disorders and history of psychiatric illness or substance abuse. Each sample was snap frozen, pulverized with small amounts of liquid nitrogen, and stored at $-80^{\circ} \mathrm{C}$ until use.

\section{Antipsychotic drug-treated rats}

The Institutional Animal Care and Use Committee of the University of Alabama at Birmingham approved all procedures using animals. Male Sprague Dawley rats (Charles River, Wilmington, MA) were intramuscularly injected every three weeks with either haloperidol decanoate in sesame oil $(28.5 \mathrm{mg} / \mathrm{kg}, N=10)$ or sesame oil only $(N=10)$ for a total of 12 injections over 9 months. The dose and duration of treatment were chosen to model chronic antipsychotic treatment in humans ${ }^{9,22}$. The animals were decapitated and brains immediately harvested, dissected, then stored at $-80^{\circ} \mathrm{C}$. Samples of rat cortex were used in this study.

\section{F-actin and G-actin fractionation}

Tissue from both human and rat samples was homogenized in F-actin stabilization buffer [0.1 M PIPES, pH 6.9, $30 \%$ glycerol, $5 \%$ dimethyl sulfoxide, $1 \mathrm{mM} \mathrm{MgSO}_{4}, 1$ mM EGTA, 1\% TritonX-100, $1 \mathrm{mM}$ adenosine triphosphate, and protease inhibitor (Complete Mini, Roche Diagnostics, Manheim, Germany)] using glass pestle tissue grinders and the protein concentration of the resulting homogenates was measured by $\mathrm{BCA}$ protein assay (Thermo Scientific, Rockford, Illinois). Samples were incubated at $37^{\circ} \mathrm{C}$ for $10 \mathrm{~min}$ and subsequently centrifuged at $2000 \mathrm{rpm}$ for $5 \mathrm{~min}$. The resulting supernatant was collected and centrifuged at $134,000 \times g$ at $37^{\circ} \mathrm{C}$ for 1 $\mathrm{h}$ in an ultracentrifuge (SW60Ti rotor, Beckman Coulter, Brea, CA) to partition G-actin (supernatant) from F-actin (pellet) fractions. The pellet was resuspended in $8 \mathrm{M}$ urea, incubated on ice for $1 \mathrm{~h}$, and vortexed every $15 \mathrm{~min}$ to depolymerize F-actin.

This protocol was adapted from and has been used extensively in cell culture studies ${ }^{23-29}$. To our knowledge, there are no existing reports of using this protocol in postmortem brain. To validate enrichment of $F$ and Gactin from brain tissue, samples from two human comparison subjects were fractionated in triplicate. The resulting fractions were treated with Phalloidin-iFluor 488 Reagent (Abcam, Cambridge, United Kingdom) at 1:500 dilution for $1 \mathrm{~h}$ at room temperature. Fluorescence was measured using a Synergy HT Microplate Reader (BioTek, Winooski, VT) and the three measures for each subject were averaged. Homogenate without phalloidin treatment was measured as a negative control. 


\section{Western blot analysis}

Samples were reconstituted in $12.5 \mu$ of ultrapure water (Milli-Q A10, Millipore, Billerica, MA) and $12.5 \mu \mathrm{l} 6 \mathrm{x}$ reducing buffer (6x solution: $5 \%$ SDS, $15 \% \beta$-mercaptoethanol, $0.0018 \%$ bromophenol blue and $36 \%$ glycerol in $170 \mathrm{mM}$ Tris- $\mathrm{HCl} \mathrm{pH} \mathrm{6.8)} \mathrm{and} \mathrm{heated} \mathrm{at} 70{ }^{\circ} \mathrm{C}$ for $10 \mathrm{~min}$. Samples were processed by sodium dodecyl sulfate polyacrylamide gel electrophoresis using Invitrogen (Carlsbad, CA) Bolt 4-12\% Bis-Tris Plus gels, electrophoresed using a Novex Bolt Mini system (Life Technologies, Grand Island, NY), and transferred onto nitrocellulose membranes (Bio-Rad, Hercules, CA) using a semi-dry transfer apparatus (Bio-Rad). Membranes were blocked with Odyssey blocking buffer (LI-COR Biosceinces, Lincoln, $\mathrm{NE}$ ) for $1 \mathrm{~h}$ at room temperature prior to incubation with anti-actin monoclonal antibody (MAB1501, Millipore) for $16 \mathrm{~h}$ at $4{ }^{\circ} \mathrm{C}$. Membranes were washed with phosphatebuffered saline (PBS) containing 0.1\% Tween-20 (PBST), and then probed with IR-Dye labeled secondary antibodies (LI-COR Biosciences). Membranes were again washed with PBST and placed in water prior to being imaged with a LI-COR Odyssey scanner. Protein bands of interest were measured using Image Studio 5.2 analytical software (LI-COR Biosciences).

The near-infrared fluorescence value for each target was normalized to the intralane value of $\beta$-tubulin, and technical replicates were averaged. $\beta$-tubulin was chosen as a loading control because it is ubiquitously expressed in human brain and was found to partition in both fractions. We found no difference in $\beta$-tubulin expression between schizophrenia and comparison groups in the G-actin fraction, F-actin fraction, or total (summed expression from G-actin and F-actin fractions), the last of which is consistent with prior reports ${ }^{10,30}$.

\section{Phalloidin-binding assay}

Tissue homogenized in F-actin stabilization buffer, as described above, containing $25 \mu$ g of protein was obtained from each subject. Triplicate samples from each subject were loaded in a 96-well plate (ThermoFisher) and treated with Phalloidin-iFluor 488 Reagent (Abcam) at 1:500 dilution for $1 \mathrm{~h}$ at room temperature. On the same plate, an equal volume of 1:500 phalloidin was placed in three wells without any sample for background measurement. Fluorescence was measured using a Synergy HT Microplate Reader (BioTek, Winooski, VT) and the average of three wells for each subject was calculated. To correct for background fluorescence in the assay, the average fluorescence of negative control wells was subtracted from each measure prior to analysis.

\section{Data analysis}

Raw total actin $\left(\mathrm{ACT}_{\text {raw }}\right)$ was calculated as the sum of average $\mathrm{F}$-actin and average G-actin expression for each subject. The standard for data analysis using this fractionation methodology is to evaluate the ratios of F-actin/Gactin, $\mathrm{F}$-actin/ACT $\mathrm{Al}_{\text {raw}}$, and $\mathrm{G}$-actin/ $/ \mathrm{ACT}_{\text {raw }}{ }^{23-29}$. These ratios represent the amount of F-actin and G-actin relative to total actin and to each other. As an additional control, we also normalized F-actin and G-actin bands to intralane $\beta$-tubulin from each sample.

Data were analyzed using Prism 6.07 software (GraphPad Software Inc., La Jolla, CA). The investigator who executed experimental protocols was blind to subject diagnosis until study completion. The D'Agostino-Pearson omnibus test was used to assess the normality of the distribution. Dependent variables not normally distributed were log transformed and verified to be normally distributed following transformation. Between group differences were assessed by two-tailed, unpaired Student's $t$-tests. For significantly different dependent measures, post hoc assessments were performed using linear regression to identify potential relationships between protein expression and subject age, tissue $\mathrm{pH}$, and postmortem interval. None of these factors demonstrated any significant association with our dependent measures. Due to the difference in sex distribution between diagnostic groups $\left(\chi^{2}=4.82, p=0.03\right)$, post hoc two-way analysis of variance (ANOVA) was performed on significantly different dependent measures to assess potential sex differences. One-way ANOVA was used to assess potential differences related to antipsychotic treatment at the time of death. For all statistical tests, $\alpha=0.05$.

\section{Results}

Validation of F- and G-actin fractionation

The fractionation technique employed has been used frequently in cell culture studies ${ }^{23-29}$, but has not yet been validated or optimized for use with samples from postmortem human brain. We measured phalloidin binding in prepared fractions to validate the enrichment of filamentous actin in the F-actin fraction, and to confirm depletion of F-actin binding in the G-actin fraction. Our results indicate substantial binding of phalloidin in the Factin fraction, consistent with enrichment of polymeric Factin in this fraction. As expected, we found no phalloidin binding in the G-actin fraction. This finding reflects a

\section{Table 2 F-actin and G-actin fractionation validation}

\begin{tabular}{lll}
\hline & Subject $\mathbf{1}$ & Subject 2 \\
\hline Phalloidin-treated F-Actin Fraction & $28 \pm 2$ & $30 \pm 2$ \\
Phalloidin-treated G-actin fraction & $7 \pm 1$ & $8 \pm 1$ \\
Untreated homogenate control & $7 \pm 1$ & $8 \pm 1$ \\
\hline
\end{tabular}

Values are relative fluorescence (arbitrary units) and reported as means \pm standard deviation of three technical replicates from each subject for each condition 

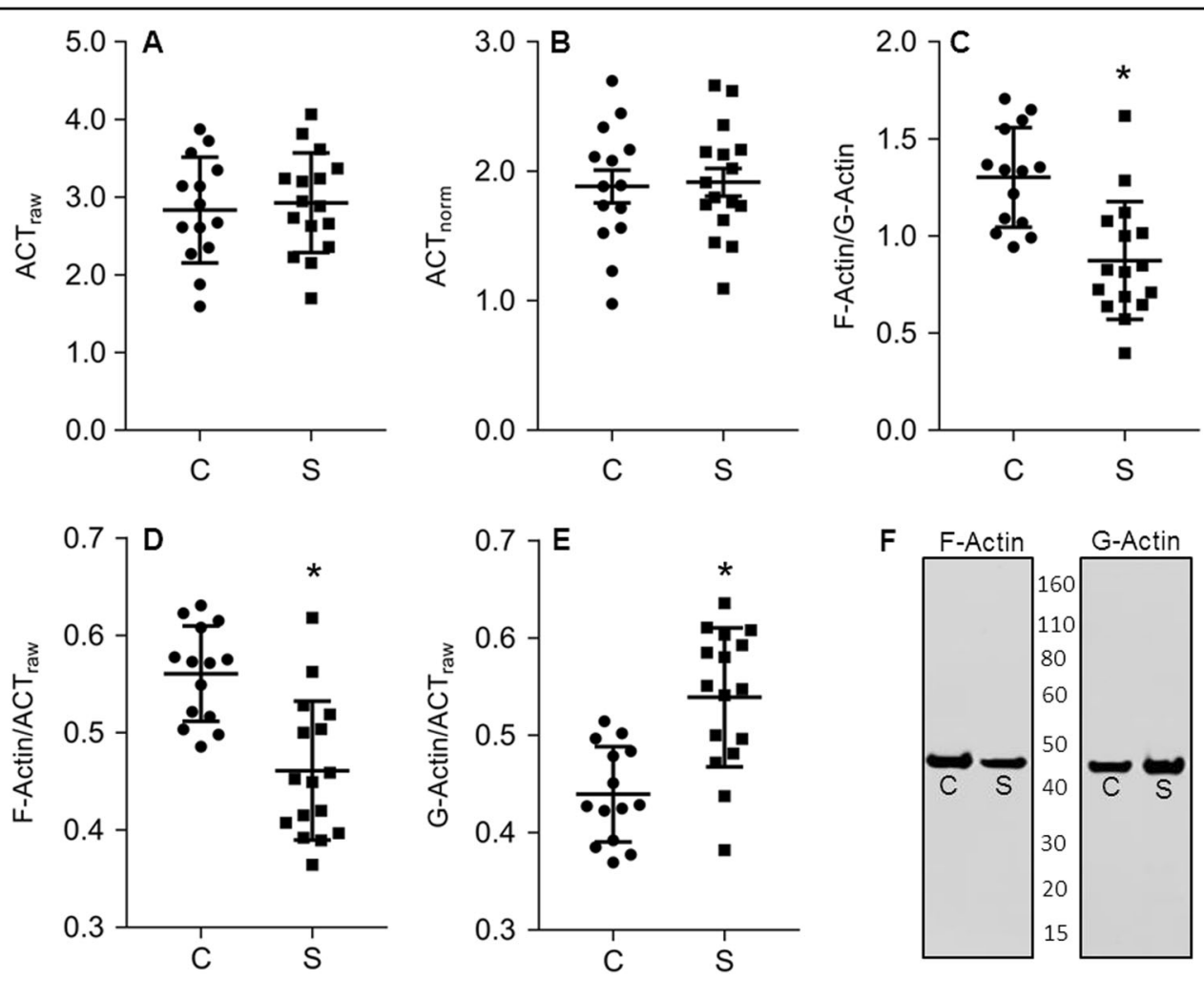

Fig. 1 Actin polymerization is reduced in schizophrenia, while total actin expression remains unchanged. a Raw total actin ( $A C T_{\text {raw }}$, the sum of the averaged $\mathrm{F}$ - and $\mathrm{G}$-actin bands, is unchanged in schizophrenia. $\mathbf{b} \beta$-tubulin-normalized total actin (ACT norm) is also unchanged in schizophrenia. $\mathbf{c}$ In schizophrenia, the ratio of $\mathrm{F}$ - to $\mathrm{G}$-actin is decreased. $\mathbf{d}$ The ratio of F-actin to $A \mathrm{CT}_{\text {raw }}$ is decreased, while $\mathbf{e}$ the ratio of $\mathrm{G}$-actin to $\mathrm{ACT}_{\text {raw }}$ increased. $\mathbf{f}$ Representative immunoblots of F-actin and $\mathrm{G}$-actin. ${ }^{*} p<0.001$

Table 3 Total actin, F-actin, G-actin, and phalloidinbinding levels in schizophrenia and comparison subjects

\begin{tabular}{lllll}
\hline & Comparison & Schizophrenia & $\begin{array}{l}\text { Test } \\
\text { statistic }\end{array}$ & p \\
& & & & \\
\hline ACT $_{\text {raw }}$ & $2.84 \pm 0.68$ & $2.93 \pm 0.64$ & $\mathrm{t}(28)=0.38$ & \\
F-actin/ACT $_{\text {raw }}$ & $0.56 \pm 0.05$ & $0.46 \pm 0.07$ & $\mathrm{t}(28)=4.39$ & 0.0001 \\
G-actin/ACT $_{\text {raw }}$ & $0.44 \pm 0.05$ & $0.54 \pm 0.07$ & $\mathrm{t}(28)=4.39$ & 0.0001 \\
F-actin/G-actin & $1.30 \pm 0.26$ & $0.89 \pm 0.07$ & $\mathrm{t}(28)=4.37$ & 0.0002 \\
ACT $_{\text {norm }}$ & $1.88 \pm 0.47$ & $1.92 \pm 0.43$ & $\mathrm{t}(28)=0.20$ & \\
F-actin/ $\beta-_{\text {tubulin }}$ & $1.05 \pm 0.25$ & $0.87 \pm 0.21$ & $\mathrm{t}(28)=2.11$ & 0.0439 \\
G-actin/ $\beta$ - & $0.83 \pm 0.24$ & $1.04 \pm 0.28$ & $\mathrm{t}(28)=2.19$ & 0.0373 \\
tubulin & & & & \\
Phalloidin (F- & $67.95 \pm 16.12$ & $47.65 \pm 15.15$ & $\mathrm{t}(28)=3.56$ & 0.0014 \\
actin) & & & & \\
\hline
\end{tabular}

Total raw actin $\left(A C T_{\text {raw }}\right), \beta$-tubulin-normalized total actin $\left(A C T_{\text {norm }}\right)$, and reported ratios are calculated as described in Methods-Data Analysis. Phalloidin (F-actin) is calculated as the average fluorescence value (arbitratry units) for each subject less the average fluorescence of negative control samples containing only phalloidin. Data are reported as means \pm standard deviation complete depletion of F-actin from the G-actin fraction in fractionated postmortem brain samples (Table 2).

\section{Decreased F-actin and increased G-actin in schizophrenia}

We found that both raw total actin $\left(\mathrm{ACT}_{\text {raw }}\right)$ and $\beta$ tubulin-normalized total actin $\left(\mathrm{ACT}_{\text {norm }}\right)$ are unchanged between diagnostic groups (Fig. 1a, b). The ratio of F/Gactin was decreased by $33 \%$ in schizophrenia (Fig. 1c). Factin/ $\mathrm{ACT}_{\text {raw }}$, a measure of the extent of actin polymerization, was decreased by $18 \%$ in schizophrenia (Fig. 1d), and $\beta$-tubulin-normalized F-actin was decreased by $17 \%$ in schizophrenia (Table 3 ). G-actin/ACT $\mathrm{A}_{\text {raw }}$ was increased by $23 \%$ (Fig. 1e), and $\beta$-tubulin-normalized Gactin expression was increased by $25 \%$ in schizophrenia relative to comparison subjects (Table 3 ). When the same dependent measures were assessed in rats chronically treated with an antipsychotic drug, none were found to be significantly different between rats treated with haloperidol and vehicle-treated controls (Table 4).

\section{Phalloidin binding is reduced in schizophrenia}

Phalloidin binding, an accepted measure of F-actin protein expression, was decreased by $30 \%$ in 
Table 4 Total actin, F-actin, G-actin, and phalloidinbinding levels in haloperidol and vehicle-treated rats

\begin{tabular}{llll}
\hline & Control & Haloperidol & Test statistic \\
\hline $\mathrm{ACT}_{\text {raw }}$ & $2.97 \pm 0.03$ & $3.01 \pm 0.03$ & $\mathrm{t}(18)=0.37$ \\
F-actin/ACT $_{\text {raw }}$ & $0.51 \pm 0.01$ & $0.50 \pm 0.02$ & $\mathrm{t}(18)=1.22$ \\
G-actin/ACT $_{\text {raw }}$ & $0.49 \pm 0.01$ & $0.50 \pm 0.02$ & $\mathrm{t}(18)=0.70$ \\
F-actin/G-actin & $0.99 \pm 0.06$ & $0.98 \pm 0.04$ & $\mathrm{t}(18)=0.31$ \\
$\mathrm{ACT}_{\text {norm }}$ & $1.97 \pm 0.06$ & $1.99 \pm 0.06$ & $\mathrm{t}(18)=0.70$ \\
F-actin/ $\beta$-tubulin & $0.98 \pm 0.03$ & $1.00 \pm 0.04$ & $\mathrm{t}(18)=1.63$ \\
G-actin/ $\beta$-tubulin & $0.99 \pm 0.06$ & $0.98 \pm 0.04$ & $\mathrm{t}(18)=0.31$ \\
Phalloidin (F-actin) & $58.27 \pm 10.61$ & $58.13 \pm 8.93$ & $\mathrm{t}(18)=0.03$ \\
\hline
\end{tabular}

Total raw actin $\left(\mathrm{ACT}_{\text {raw }}\right), \beta$-tubulin-normalized total actin $\left(\mathrm{ACT}_{\text {norm }}\right)$, and reported ratios are calculated as described in Methods-Data Analysis. Phalloidin (F-actin) is calculated as the average fluorescence value (arbitratry units) for each subject less the average fluorescence of negative control samples containing only phalloidin. Data are reported as means \pm standard deviation; no variables were significantly different between treatment conditions

schizophrenia relative to non-psychiatrically ill comparison subjects (Table 3). Phalloidin binding was unchanged in antipsychotic-treated rats (Table 4).

\section{Discussion}

In this study, we measured the extent of actin polymerization in the anterior cingulate cortex of schizophrenia and comparison subjects using both an F-actin/ G-actin fractionation technique and the selectively Factin-binding protein phalloidin. Western blot analysis of F-actin and G-actin fractions revealed decreased expression of F-actin when assessed independently and relative to total actin expression. Treatment of subjects with fluorescently-labeled phalloidin also independently revealed decreased expression of F-actin. In conjunction with a reduced F/G-actin ratio, these findings are consistent with our hypothesis that actin polymerization is impaired in schizophrenia.

Actin is a crucial component of cellular scaffolding responsible for establishing and maintaining cell morphology, and actin dynamics underlie core cellular processes including cell motility and intracellular protein trafficking ${ }^{31}$. In developing neurons, actin remodeling is vital to synaptogenesis and in the formation, extension, and development of neurites. In mature neurons, actin is the most abundant cytoskeletal protein at synapses and is present at both pre- and postsynaptic terminals. Modulation of actin dynamics is necessary for the cytoarchitectural changes that accompany synaptic plasticity ${ }^{32-35}$.

Actin polymerization is stimulated by nucleating factors, the most prominent of which is the Arp $2 / 3$ complex $^{36}$. Interestingly, Arp2/3 knock-out mice exhibit many hallmark neurobiological traits of schizophrenia, including decreased spine formation, enhanced excitation of cortical neurons, and abnormal striatal output, suggesting dysfunction of the complex in this illness ${ }^{16}$. Knockdown of cortactin and N-WASP, NPFs, and activators of the Arp2/3 complex, results in decreased dendritic spine density in hippocampal neurons ${ }^{37,}{ }^{38}$. Multiple lines of evidence suggest reduced activity of the Arp2/3 complex in schizophrenia ${ }^{18,19}$, which could in turn diminish actin polymerization, as our current findings suggest.

Converging evidence has implicated synaptic dysfunction and reduced dendritic spine density as key components in the pathophysiology of schizophrenia ${ }^{1-5,39}$. It is thought that enlargement of dendritic spines is facilitated by an increase in the F-actin/G-actin ratio, while reduced spine size is associated with a decreased F-actin/G-actin ratio $^{40,}{ }^{41}$. Reduced actin polymerization indicates impaired actin nucleation and/or cross-linking of F-actin filaments, processes which are critical for the activitydependent structural plasticity of spines, which can contribute to decreased spine stability and ultimately spine loss ${ }^{13}$. Decreased cortical dendritic spine density has been hypothesized to be associated with hyperdopaminergia in schizophrenia ${ }^{19}$. Our findings of decreased F-actin and increased G-actin in schizophrenia (Fig. 1, Table 3) are consistent with this model, and suggest a mechanism that contributes to the reduced dendritic spine density and spine length in the illness. Enhancing actin polymerization might increase the density and length of dendritic spines, and thus, targeted modulation of actin polymerization in brain may be an exciting avenue for further exploration.

There are some limitations to this study in postmortem human brain. The patients studied were elderly and in late stages of the illness, and these findings may not generalize to younger patients. Another potential limitation of the current study is that the ratio of male:female subjects was different between diagnostic groups. To account for this, we conducted post hoc two-way ANOVA for all significantly different dependent measures using sex and diagnosis as the independent variables. We did not identify any effect of sex on protein expression.

Potential effects of chronic antipsychotic treatment on protein expression are also a concern in schizophrenia studies. To address the possibility that changes in actin polymerization status may be due to schizophrenia subjects receiving chronic antipsychotic treatment, we conducted parallel experiments in rats chronically treated with haloperidol. Protein expression of F-actin, G-actin, $\mathrm{ACT}_{\text {raw }}, \mathrm{ACT}_{\text {norm, }}$ and ratios of actin forms, as well as phalloidin binding, were not different between haloperidol and vehicle-treated rats (Table 4). We also performed post hoc statistical analyses for all significantly different dependent measures between schizophrenia subjects who received antipsychotic treatment within 6 weeks of death vs. those who had been off medication for at least 6 weeks prior to death. No medication effects were identified via 
post hoc statistical analyses nor by alterations in an antipsychotic-treated rat model. Thus, it is unlikely that the abnormalities reported here are due to chronic medication effects, and these findings are more likely associated with the illness itself.

We identified reduced protein expression of F-actin in schizophrenia using converging techniques, as well as increased G-actin expression and reduced F/G-actin ratio. These findings are consistent with decreased actin polymerization, and suggest a mechanistic explanation for altered dendritic spine morphology and abundance observed in schizophrenia. Dendritic spines are the prime recipients of excitatory synaptic input and decreased dendritic spine density is known to impair synaptic plasticity, which may contribute to cognitive dysfunction associated with schizophrenia. These data implicate decreased actin polymerization as a potential substrate of the pathophysiology of this illness and may suggest actin remodeling as a potential target for novel treatments.

\section{Acknowledgements}

This work is supported by National Institutes of Health Grant MH53327 (J.H.M.W.), MH064673 (VH) and MH066392 (VH). We gratefully acknowledge Bronx VA and Mount Sinai Center Brain Bank for providing tissue samples. Also, we would like to acknowledge the Alabama Brain Collection.

\section{Competing interests}

The authors declare that they have no competing financial interests.

Publisher's note: Springer Nature remains neutral with regard to jurisdictional claims in published maps and institutional affiliations.

Received: 25 May 2017 Revised: 13 September 2017 Accepted: 15 September 2017

Published online: 11 December 2017

\section{References}

1. Lewis, D. A., Glantz, L. A., Pierri, J. N. \& Sweet, R. A. Altered cortical glutamate neurotransmission in schizophrenia: evidence from morphological studies of pyramidal neurons. Ann. N. Y. Acad. Sci. 1003, 102-112 (2003).

2. Glantz, L. A. \& Lewis, D. A. Dendritic spine density in schizophrenia and depression. Arch. Gen. Psychiatr. 58, 203 (2001)

3. Garey, L. J. et al. Reduced dendritic spine density on cerebral cortical pyramidal neurons in schizophrenia. J. Neurol. Neurosurg. Psychiatr. 65, 446-453 (1998).

4. Glantz, L. A. \& Lewis, D. A. Decreased dendritic spine density on prefrontal cortical pyramidal neurons in schizophrenia. Arch. Gen. Psychiatr. 57, 65-73 (2000).

5. Glausier, J. R. \& Lewis, D. A. Dendritic spine pathology in schizophrenia. Neuroscience 22, 90-107 (2013).

6. Fatemi, S. H., Stary, J. M., Earle, J. A., Araghi-Niknam, M. \& Eagan, E. GABAergic dysfunction in schizophrenia and mood disorders as reflected by decreased levels of glutamic acid decarboxylase 65 and $67 \mathrm{kDa}$ and Reelin proteins in cerebellum. Schizophr. Res. 72, 109-122 (2005)

7. Chai, X., Forster, E., Zhao, S., Bock, H. H. \& Frotscher, M. Reelin stabilizes the actin cytoskeleton of neuronal processes by inducing $n$-cofilin phosphorylation at serine3. J. Neurosci. 29, 288-299 (2009).

8. Hayashi-Takagi, A. et al. Disrupted-in-Schizophrenia 1 (DISC1) regulates spines of the glutamate synapse via Rac1. Nat. Neurosci. 13, 327-332 (2010).

9. Rubio, M. D., Haroutunian, V. \& Meador-Woodruff, J. H. Abnormalities of the Duo/Ras-related C3 botulinum toxin substrate 1/p21-activated kinase 1 pathway drive myosin light chain phosphorylation in frontal cortex in schizophrenia. Biol. Psychiatr. 10, 906-914 (2012).

10. Bauer, D. E., Haroutunian, V., McCullumsmith, R. E. \& Meador-Woodruff, J. H. Expression of four housekeeping proteins in elderly patients with schizophrenia. J. Neural Transm. Nienna, Austria: 1996) 116, 487-491 (2009).

11. Fatemi, S. H., Folsom, T. D. \& Thuras, P. D. Deficits in GABA(B) receptor system in schizophrenia and mood disorders: a postmortem study. Schizophr. Res. 128, 37-43 (2011)

12. Smart, F. M. \& Halpain, S. Regulation of dendritic spine stability. Hippocampus 10, 542-554 (2000).

13. Matus, A. Actin-based plasticity in dendritic spines. Science 290, 754-758 (2000).

14. Hotulainen, P. \& Hoogenraad, C. C. Actin in dendritic spines: connecting dynamics to function. J. Cell Biol. 189, 619-629 (2010).

15. Lewis, D. A. \& Gonzalez-Burgos, G. Pathophysiologically based treatment interventions in schizophrenia. Nat. Med. 12, 1016-1022 (2006).

16. Kim, I. H. et al. Spine pruning drives antipsychotic-sensitive locomotion via circuit control of striatal dopamine. Nat. Neurosci. 18, 883-891 (2015).

17. Pollard, T. D. Regulation of actin filament assembly by Arp $2 / 3$ complex and formins. Annu. Rev. Biophys. Biomol. Struct. 36, 451-477 (2007)

18. Bhambhvani, H. P., Simmons, M., Haroutunian, V. \& Meador-Woodruff, J. H. Decreased expression of cortactin in schizophrenia brain. Neuroreport 27, 145-150 (2016)

19. Datta, D., Arion, D., Roman, K. M., Volk, D. W. \& Lewis, D. A. Altered expression of ARP2/3 complex signaling pathway genes in prefrontal layer 3 pyramidal cells in schizophrenia. Am. J. Psychiatr. 174, 163-171 (2017).

20. Rubio, M. D., Wood, K., Haroutunian, V. \& Meador-Woodruff, J. H. Dysfunction of the ubiquitin proteasome and ubiquitin-like systems in schizophrenia. Neuropsychopharmacology 38, 1910-1920 (2013).

21. Mueller, T. M., Remedies, C. E., Haroutunian, V. \& Meador-Woodruff, J. H. Abnormal subcellular localization of GABAA receptor subunits in schizophrenia brain. Transl. Psychiatr. 5, e612 (2015).

22. Kippe, J. M., Mueller, T. M., Haroutunian, V. \& Meador-Woodruff, J. H. Abnormal $\mathrm{N}$-acetylglucosaminyltransferase expression in prefrontal cortex in schizophrenia. Schizophr. Res. 166, 219-224 (2015)

23. Caution, K., Gavrilin, M. A. \& Tazi, M. et al. Caspase-11 and caspase-1 differentially modulate actin polymerization via RhoA and Slingshot proteins to promote bacterial clearance. Sci. Rep. 5, 18479 (2015).

24. Huang, W., Zhu, P. J. \& Zhang, S. et al. mTORC2 controls actin polymerization required for consolidation of long-term memory. Nat. Neurosci. 16, 441-448 (2013).

25. Heacock, C. S. \& Bamburg, J. R. The quantitation of G- and F-actin in cultured cells. Anal. Biochem. 135, 22-36 (1993).

26. Katsantonis, J. et al. Differences in the $\mathrm{G} /$ total actin ratio and microfilament stability between normal and malignant human keratinocytes. Cell Biochem Funct. 12, 267-274 (1994).

27. Rasmussen, I. et al. Effects of F/G-actin ratio and actin turn-over rate on NADPH oxidase activity in microglia. BMC Immunol. 11, 44 (2010).

28. Han, Yu, Wang, Xianren, Chen, Jun \& Sha, Su-Hua Noise-induced cochlear Factin depolymerization is mediated via ROCK2/p-ERM signaling. J. Neurochem. 133, 617-628 (2015)

29. Theodoropoulos, P. A. et al. Hepatocyte swelling leads to rapid decrease of the $\mathrm{G}$-/total actin ratio and increases actin mRNA levels. FEBS Lett. 311, 241-245 (1992).

30. Tucholski, J. et al. Abnormal N-linked glycosylation of cortical AMPA receptor subunits in schizophrenia. Schizophr. Res. 146, 177-183 (2013).

31. Dominguez, R. \& Holmes, K. C. Actin structure and function. Ann. Rev. Biophys. 40, 169-186 (2011).

32. Landis, D. M., Hall, A. K., Weinstein, L. A. \& Reese, T. S. The organization of cytoplasm at the presynaptic active zone of a central nervous system synapse. Neuron 1, 201-209 (1988).

33. Bloom, O., Evergren, E. \& Tomilin, N. et al. Colocalization of synapsin and actin during synaptic vesicle recycling. J. Cell Biol. 161, 737-747 (2003).

34. Matus, A., Ackermann, M., Pehling, G., Byers, H. R. \& Fujiwara, K. High actin concentrations in brain dendritic spines and postsynaptic densities. Proc. Natl Acad. Sci. U.S.A. 79, 7590-7594 (1982)

35. Cingolani, L. A. \& Goda, Y. Actin in action: the interplay between the actin cytoskeleton and synaptic efficacy. Nat. Rev. Neurosci. 9, 344-356 (2008).

36. Goley, E. D. \& Welch, M. D. The ARP2/3 complex: an actin nucleator comes of age. Nat. Rev. Mol. Cell Biol. 7, 713-726 (2006). 
37. Hering, H. \& Sheng, M. Activity-dependent redistribution and essential role of cortactin in dendritic spine morphogenesis. J. Neurosci. 23, 11759-11769 (2003).

38. Wegner, A. M., Nebhan, C. A. \& Hu, L. et al. N-WASP and the Arp2/3 complex are critical regulators of actin in the development of dendritic spines and synapses. J. Biol. Chem. 283, 15912-15920 (2008).

39. McGlashan, T. H. \& Hoffman, R. E. Schizophrenia as a disorder of developmentally reduced synaptic connectivity. Arch. Gen. Psychiatr. 57, 637-648 (2000).
40. Fukazawa, Y. et al. Hippocampal LTP is accompanied by enhanced F-actin content within the dendritic spine that is essential for late LTP maintenance in vivo. Neuron 38, 447-460 (2003).

41. Zhou, Q., Homma, K. J. \& Poo, M. M. Shrinkage of dendritic spines associated with long-term depression of hippocampal synapses. Neuron 44, 749-757 (2004). 